\title{
S7. Psychological diseases in general care
}

Psychological Problems in General Health Care:

WHO Collaborative Project

T.B. Ustün on behalf of all the Participating Investigators *

World Health Organization, Division of Mental Health, Geneva, Switzerland

OBJECTIVES: This paper reports the general design and findings from a WHO collaborative study of the "Psychological Problems in General Health Care".

METHODS: This project was carried out in 14 countries in different parts of world. Research methods are described in detail in Arch Gen Psychiatry Vol 50, pp 819-824, Oct 1993.

STUDY RESULTS: Psychological problems are frequent in all the general health care settings examined in this study i.e depressive disorders, anxiety disorders, alcohol use disorders, and somatoform disorders. All these disorders were associated with marked disability. The concordance between clinician recognition of psychological disorder and the research diagnosis of definite ICD-10 psychological disorder was low to moderate. Hence the patients with psychological problems were neither recognized, nor treated sufficiently.

CONCLUSION: The psychological disorders of general health care patients constitute a major public health problem worldwide because: they are frequent; can have severe consequences for individuals and societies; and existing specific and acceptable treatments are not currently being applied to a sufficient degree.

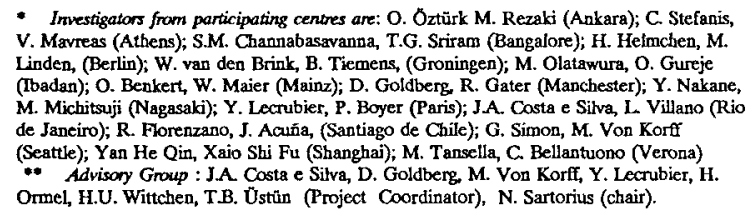

Pathways to General Health Care of Patients with Psychiatric Disonder

R A. Gater, D.P. Goldberg, M. Rezaki, M. Tansella, Yan He Qin, L. Villano, T.B. Üstün. N. Sartorius and the Principal Investigators of the Participating Centres.

OBJECTIVES: This paper reports findings from a WHO collaborative study of the "Psychological Problems in General Health Care". particularly on how mentally ill patients reach to primary care and compares it with the pathways to specialist care.

METHODS: This study examines the referral pathways by which patients with mental disorders reach primary care physicians. It investigates the associations between recognition of psychiatric disorder by the primary care physicians and the pathways taken by patients seeking care with psychiatric disorder. The research methods employed are described in detail in Arch Gen Psychiatry Vol 50, pp 819-824, Oct 1993.

STUDY RESULTS: The results demonstrate that the ability of primary care physicians to detect mental disorders is closely related to the pathways into primary care. In those centres where primary care physicians were well able to detect disorders, prevalence of disorder tended to be higher and most patients went directly from the community tot he primary care physician, with few seeking care elsewhere. However, in those centres where recognition by the primary care physician was poor, prevalence was lower, more patients had previously sought care from other carers in the community including other primary care physicians, and there was an important direct pathway to specialist mental health care which by-passed the primary care physician.

CONCLUSION: In Pathways to Mental Health Services,primary care physicians and their recognition of mental disorders form an important step. 
Psychological Problems in Primary Care: Presentation and Diagnosis

Y. Lecrubier, D. Goldberg, T. B. Üstün, N. Sartorius and the Investigators of the Participating Centers

OBJECTIVES: This report explores (1) the form and frequency of ICD-10 mental disorders in different participating centres (2) the effects of different levels of caseness (i.e. well-defined, subthreshold, common symptom patterns)

METHODS: The research methods employed are described in detail in Arch Gen Psychiatry Vol 50, pp 819-824, Oct 1993. Using standard diagnostic algorithms for ICD definitions for mental disorders, prevalence rates were calculated for current (one-month) and lifetime disorders on the basis of CIDI Primary Health Care Version.

STUDY RESULTS: Patients with a formal ICD-10 diagnosis are frequent in primary care: their estimated prevalence among consecutive attenders is $24 \%$. Still, the reason given for visit is psychological only for $5.3 \%$, pain for $29.3 \%$ and physical illness for $34.8 \%$.

The commonest disorders are depressive disorders (11.7), anxiety disorders (10.1), somatoform disorders (8.1) and alcohol disorders (6.0). Patients with a sub-threshold disorder missing one criterion are about $10 \%$. These patients had clinically significant symptoms and a substantial impairment. There were differences across centres in prevalence rates for most disorders but the figures obtained are consistent with prior finding in the corresponding countries. Comorbidity was frequent ( 9.5 with two or more, 14.5 with one diagnosis).

CONCLUSION: Important findings were consistently replicated across the participating centres which differed markedly in organization of care, patient population and culture. Psychological disorders were common among general health care patients in both developed and developing countries.

Impact of the Primary Care Mental Disorders: Disability and Service Utilization.

\section{J. Omel, M. Von Korff, T.B. Ustün, N. Sartorius and the Principal} Investigators of the participating centres.

OBJECTIVES: This report assesses the replicability of the relationship between depression and disability among primary care patients in an international, multi-centre study.

METHODS: This paper reports findings of the data collection methods from a WHO collaborative study of the "Psychological Problems in General Health Care". The research methods employed are described in detail in Arch Gen Psychiatry Vol 50, pp 819-824, Oct 1993.

STUDY RESULTS: The results show that the level of disability was strongly associated with psychiatric status. Pooled across all centers $23.3 \%$ of the patients with a definite disorder had moderate disability and an additional $13.1 \%$ had severe disability (all estimates are provisional based on weighted data). Among patients without a subthreshold disorder, $17.3 \%$ had moderate disability and an additional $5.7 \%$ were severely disabled. In contrast, among patients without a definitc or subthreshold disorder, $10.3 \%$ were moderately disabled and $2.5 \%$ were severely disabled. This relationship held for all of the centres, although the strength of the association varied. Among patients with major depression $(n=1122), 25.9 \%$ had moderate disability and an additional $16.4 \%$ had severe disability. The pooled estimate of the correlation between the number of depressive symptoms and the three disability measures was $r=0.29$ for Activity Limitation Days.

CONCLUSION: Across diverse countries and cultures, the presence of psychological disorder was associated with substantial levels of disability among patients studies in general health care settings.
The Treatment of Mental Disorders in Primary Health Care: Results of an International Study.

M. Linden, C. Bellantuono, V. Mavreas, $O$. Benkert and $G$. Simon and Principal Investigators of the participating centres.

OBJECTIVES: This paper deals with the question of treatment of common mental disorders in primary health care settings.

METHODS: In an international study of WHO on "Psychological Problems in Primary Health Care", 5604 patients have been assessed in 15 centres around the world. Addition to a thorough assessment of their health status, patients and physicians have also been asked about the treatment for mental disorders. The research methods employed are described in detail in Arch Gen Psychiatry Vol 50, pp 819-824, Oct 1993.

STUDY RESULTS: The results show that on an average $10 \%$ of all patients, $53 \%$ of patients with a physician diagnosis of mental disorder, and $24 \%$ of ICD/CIDI-diagnosed cases get drug treatment because of mental disorders. This rate varies considerably between centers and for different diagnostic groups. Large variations are also seen in respect to drug classes. Psychological treatment is common in the sense of counselling. The rate of referrals to mental health professionals depends on the type of disorder with $7 \%$ in anxiety or $28 \%$ in psychotic disorders.

CONCLUSION: The treatment of psychological disorders in general health care settings were provided as counselling, sedative medications, and anti-depressant medications regardless of the diagnostic status of the patient. The type of treatment did not show any association with the research diagnosis of the patients. Centres differed in their preferred treatments. 